\title{
Study on Low-Frequency TEM Effect of Coal during Dynamic Rupture
}

\author{
Cheng-wu Li, Chuan Wang, Bei-jing Xie, Xiao-yuan Sun, and Xiao-meng Xu \\ School of Resource and Safety Engineering, China University of Mining and Technology (Beijing), Beijing 100083, China \\ Correspondence should be addressed to Chuan Wang; wangchuan010bj@163.com and Bei-jing Xie; bjxie1984@163.com
}

Received 24 June 2014; Revised 29 August 2014; Accepted 12 September 2014

Academic Editor: Caiping Lu

Copyright (C) 2015 Cheng-wu Li et al. This is an open access article distributed under the Creative Commons Attribution License, which permits unrestricted use, distribution, and reproduction in any medium, provided the original work is properly cited.

\begin{abstract}
Dynamic loads provided by the SHPB test system were applied to coal specimens, and the TEM signals that emerged during coal rupture were recorded by the TMVT system. Experiments on coal-mass blasting rupture in excavating workface were also carried out, and the emerged TEM signal was analyzed. The results indicate that the low-frequency TEM signals were detected close to the coal specimens under high strain dynamic load applied by the SHPB, initially rising sharply and dropping rapidly, followed by a small tailing turbulence. And the field test results obtained during coal blasting process coincided with the results from the SHPB tests. Furthermore, its initial part shaped like a pulse cluster had a more pronounced tail and lasted even longer. And the generation mechanism of the low-frequency TEM effect was analyzed. It suggests that the low-frequency TEM effect of coal during dynamic rupture is contributed by the fractoemission mechanism and the resonance or waveguide effects. Because its wavelength is longer than the higher ones, the low-frequency TEM has a good anti-interference performance. That can expand the scope and performance of the coal-rock dynamic disaster electromagnetic monitoring technique.
\end{abstract}

\section{Introduction}

In deep mining engineering, coal and rock masses have always been exposed to intense dynamic loading, while dynamic disasters such as rock burst, coal and gas outburst can occur [1-3]. The destruction of the coal-rock structure reveals features consistent with quick rupture at high strain rates. This is reflected in significant characteristics of nonlinear dynamics, accompanied by sound, electricity, magnetism, vibration, and other physical effects [4-8]. The phenomenon of electromagnetic radiation (EMR) is considered to be closely related to the deformation and fracture of coal or rock under load $[9,10]$. Also the transient electromagnetic (TEM) effect near to coal subjected to dynamic loads has been initially confirmed [11-13]. Therefore, EMR has become a very effective method for coal-rock activity monitoring [14-16].

Studies on EMR produced during the process of coalrock deformation or fracture show that the frequency of EMR has a wide bandwidth. The main frequency band is variable $[17,18]$. EMR signals of different frequencies are generated by different mechanisms and have different waveforms.
Currently, the EMR detectors [19] for detecting coal and rock dynamic disasters were developed on the basis of EMR of medium-high-frequency ( $\geq 5 \mathrm{kHz})$. But in actual mining environments, the wavelength of medium-high-frequency EMR signal is too short to propagate more than $20 \mathrm{~m}$. Also it is vulnerable to the high-frequency interference from mechanical and electrical equipment [20]. However, low-frequency electromagnetic wave can propagate a longer distance (around $3.5 \mathrm{~km}$ ), with a small attenuation [21], and will be less affected by mining equipment. So, it is very important to study the characteristics of low-frequency EMR from coal under dynamic loading, to develop monitoring and early warning technology for coal and rock mines.

On the basis of previous work, this paper focuses on low-frequency TEM effect that emerges during coal rupture under dynamic loading. Considering the high strain rate of dynamic loading, mainly obtained by using SHPB [22] or blasting process [14], the methods of SHPB and in situ blasting tests were adopted to obtain the TEM signals during coal rupture. By combining basic theories such as charge separation, transfer theory $[23,24]$, and coal structure 


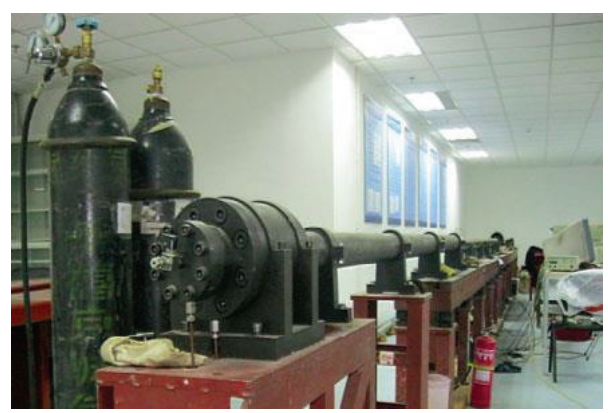

(a) SHPB test system and device

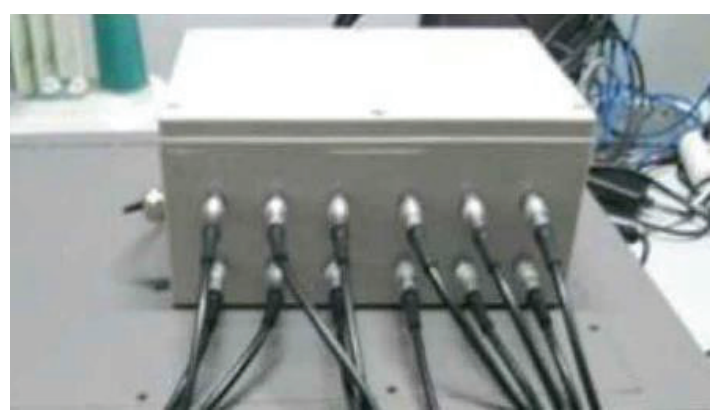

(b) ZDKT-1-type TMVT system

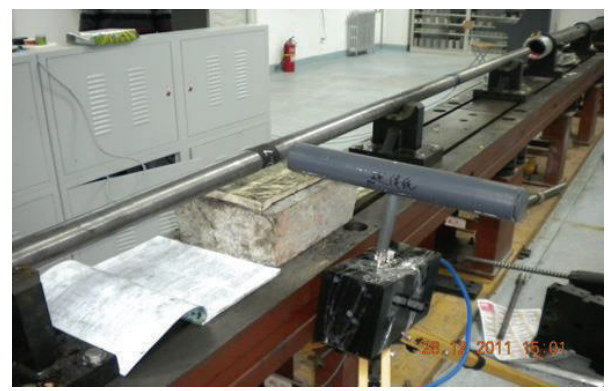

(c) Location of the TEM receiver (antenna)

FIgURE 1: Test systems and their location.

resonance theory [25], the characteristics of the TEM effect and its generation mechanism are analyzed and presented by the following sections of this paper.

\section{Experiments on TEM Effect of Coal Rupture under Dynamic Load}

2.1. Experimental Systems and Specimen. The setup used in the experiment was based on the SHPB test system and the TMVT system.

Coal impact damage experiments under dynamic load were performed on the $\Phi 50 \mathrm{~mm}$ SHPB test system in the China University of Mining (Beijing) Rock Dynamics Laboratory. Before the test, grease lubrication was applied to the interface between the elastic rod and the specimen to reduce the interfacial friction effect. Meanwhile, the ZDKT-1-type transient magnetic vibration test (TMVT) system was placed at $4 \mathrm{~cm}$ from the specimen, so as to record the TEM signal. The SHPB test system and the ZDKT-1-type TMVT system and devices used in the experiment are shown in Figure 1.

The SHPB test system contains the following: an impacting rod, two pressure bars, a gun system, ultradynamic strain gauges, and a data storage system. The impactor is a $\Phi 50 \mathrm{~mm} \times 400 \mathrm{~mm}$ round steel rod, and the impact velocity is measured by a velocimeter. The TMVT system contains the following: an antenna (internal resistance is $21.8 \mathrm{ohms}$ ), a signal amplifier, and a data acquisition recorder.

The specimens tested in the test were obtained from a lump of coal from the excavating workface of number 10 mine of Pingdingshan Coal Group Co., Ltd. According to the optimum length-diameter ratio formula [26], cut cylindrical
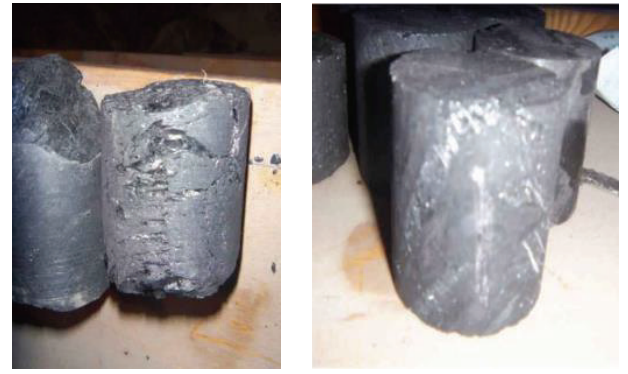

FIgURE 2: Coal specimens.

samples of $50 \mathrm{~mm}$ in diameter and $100 \mathrm{~mm}$ in length (of 2 times diameter) for SHPB test. The coal specimens are shown in Figure 2.

2.2. Experiment of TEM Signals during Coal Deformation under Dynamic Impact Load. Before the test, the SHPB test system should be adjusted [27] to meet the Stress Equilibrium principle. 14 specimens were tested at impact velocities of $4.174,4.534,5.001,6.709,6.951,8.714,9.004,10.700,12.730$, $12.746,14.503,14.847,17.559$, and $17.652 \mathrm{~m} / \mathrm{s}$.

During the experiment, the TEM signals were collected by the TMVT system. Some test results are shown in Figure 3.

From Figure 3, all the electromagnetic signals present abrupt part, with a short duration. They also contain a lot of noise which may come from the environment or the system itself. 

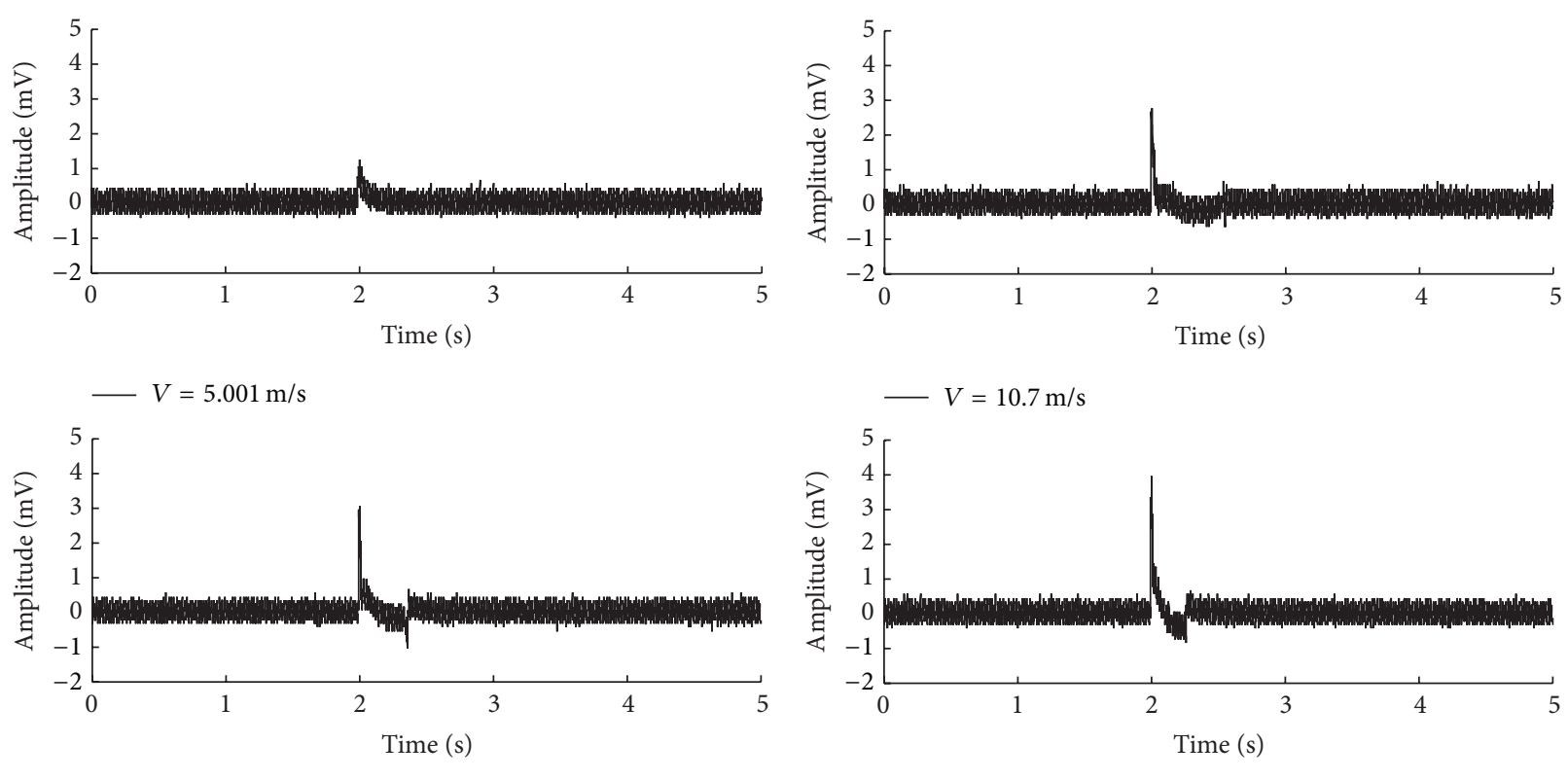

$-V=14.847 \mathrm{~m} / \mathrm{s}$

$-V=19.361 \mathrm{~m} / \mathrm{s}$

Figure 3: Original signals.
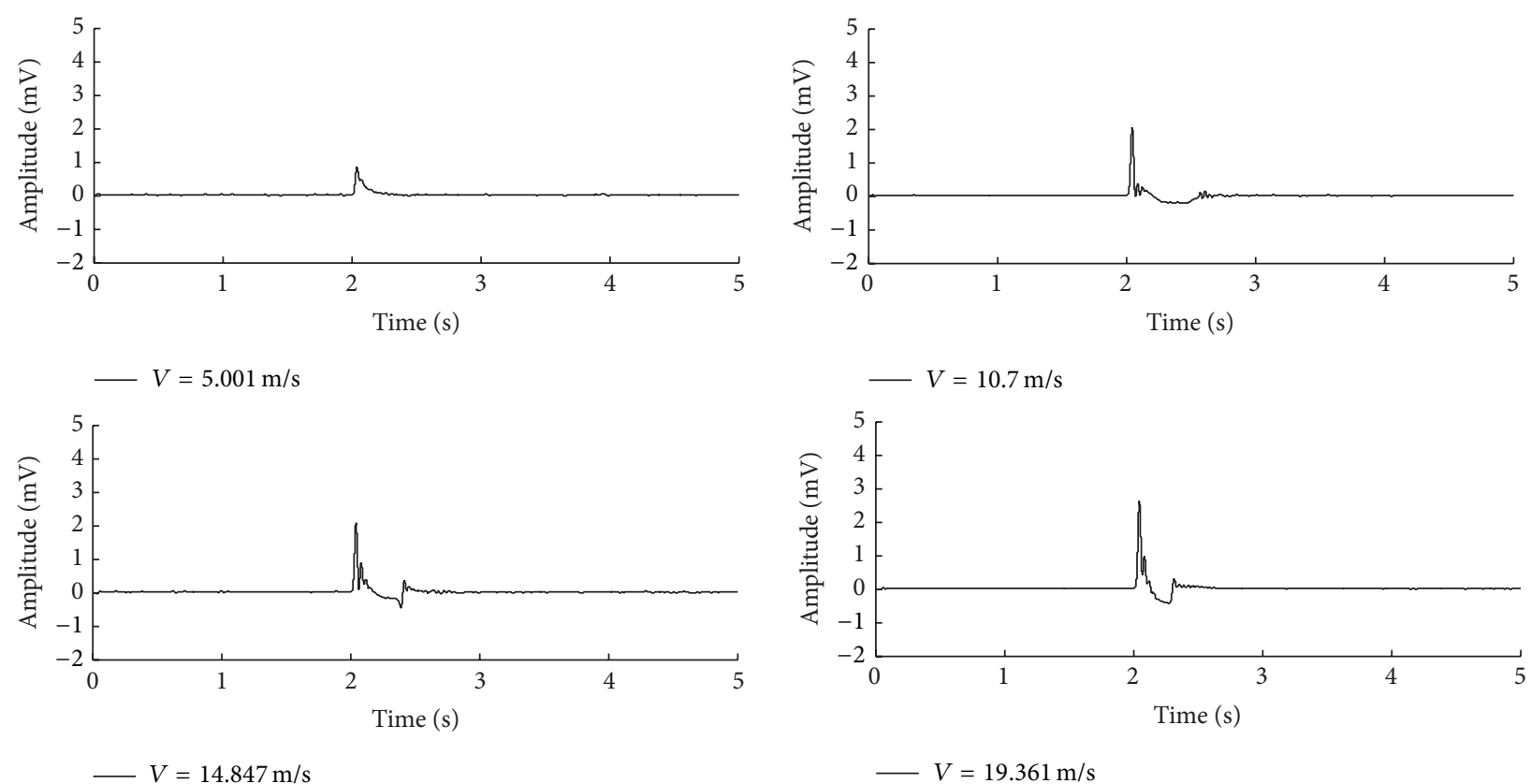

Figure 4: Processed signals.

In order to obtain a more realistic representation of the TEM signals, Data-Demon software was used to process them. The results are shown in Figure 4.

Figure 4 shows that, when processed by the "ensemble empirical mode decomposition" (EEMD) method [28], the noise in the recorded signals was filtered out, and then the true TEM signal was clearly retained. The true TEM signals rose quickly and then decreased rapidly with a slight tailing oscillation. FFT spectrum analysis results are shown in Figure 5.
Figure 5 shows that the TEM signal emerged during SHPB impact of coal as a low-frequency signal, with frequencies less than $40 \mathrm{~Hz}$.

2.3. Analysis and Discussion. The peak values (maximum amplitude) of each signal were obtained (see Figure 6). The maximum amplitude of the signal can be seen to increase linearly with the impact velocity.

But that can be obviously divided into three regions. (1) When the impact velocity is small (under $5 \mathrm{~m} / \mathrm{s}$ ), the peak 


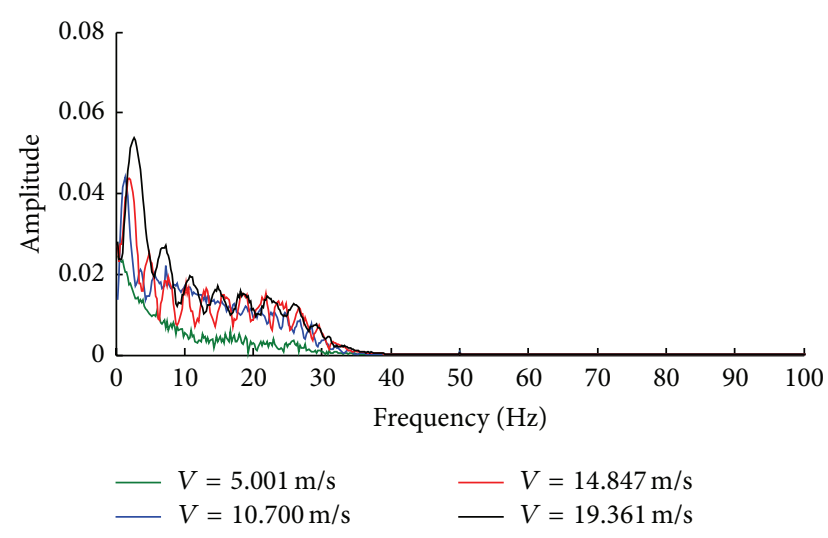

FIGURE 5: FFT spectrum of some signals.

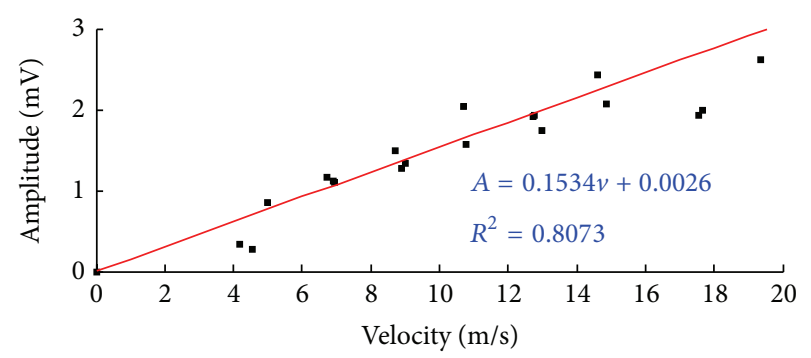

FIGURE 6: Relationship between the true TEM peak value and the impact velocity.

value is smaller than that given by the line. (2) While at the medium velocity $(5 \sim 15 \mathrm{~m} / \mathrm{s})$, the peak value has a very good linear relationship with the velocity. (3) At the high velocity (greater than $15 \mathrm{~m} / \mathrm{s}$ ), the peak value also lies under the line.

That means, in the low velocity stage, the peak value increases quickly with velocity, and in the high velocity stage, the peak value grows more slowly. Since less data was obtained at high velocity, whether the peak value continues to increase or not, this needs further research.

The energy of the TEM signal can be described by

$$
E=\frac{U^{2}}{R}=\frac{1}{R} \sum_{i=1}^{N} u_{i}^{2},
$$

where $E$ is the energy of the TEM signal, $R$ is the internal resistance, $R=21.8 \Omega$, and $U$ is the induced electromotive force, equal to the immediate detected value, $\mathrm{mV}$.

A quadratic fit to the data is presented in Figure 7.

It can be seen that a quadratic relationship has a similar pattern as the linear one as shown in Figure 6. At the high velocity, the energy data deviates significantly.

\section{Field Test of TEM Signals during Coal Rupture under Blasting Shock}

The ZDKT-1-type transient magnetic vibration detection (TMVD) system was set up in the 21180\# excavating workface, in number 10 mine of Pingdingshan Coal Group, to carry

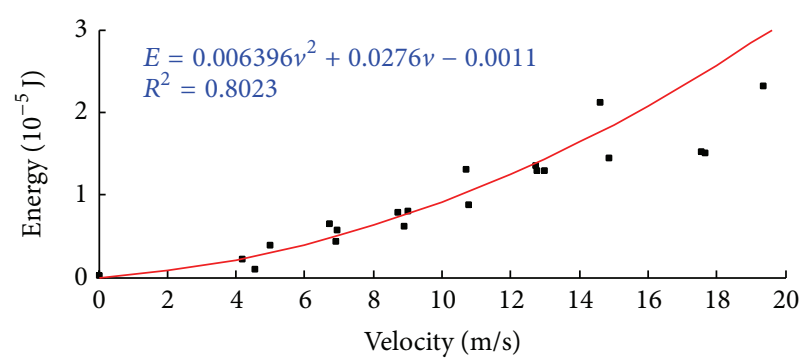

FIGURE 7: Relationship between the true TEM energy and the impact velocity.

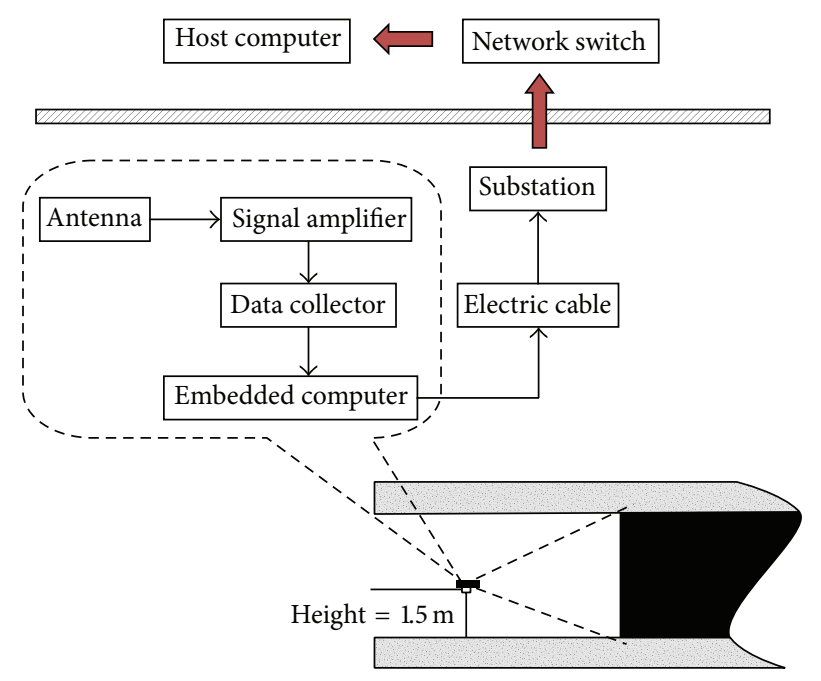

Figure 8: The field test TMVD system diagram.

out a field test of TEM produced by large-scale coal-mass dynamic rupture under actual blasting loads.

3.1. Field Test System. The TMVD system consists of a TEM detector, an electric cable, a substation of monitoring (underground), and a network switch and a host computer (above ground). The detector consists of a TEM signal receiving antenna, a signal amplifier, and a data acquisition card.

The detector was placed $300 \mathrm{~m}$ away from the tunnel opening (during the test period it remains about $200 \mathrm{~m}$ away from the workface). The TEM signal produced during coal blasting rupture was received by the antenna, converted into an electrical signal, which was amplified by the signal amplifier, converted into a digital signal via the data acquisition card, and, finally, transmitted to the ground host computer by an underground cable.

Figure 8 is a schematic diagram of the field test system.

3.2. Field Test Result Analysis and Discussion. 22 blasting tests were performed in the field. Figure 9 shows a typical TEM signal collected by the system during blasting excavating.

It can be seen from Figure 9 that the TEM signal obtained is nonstationary, is of weak strength, and contains a lot of noise. A more distinct signal was extracted from the original signal by the EEMD method, as shown in Figure 10. 


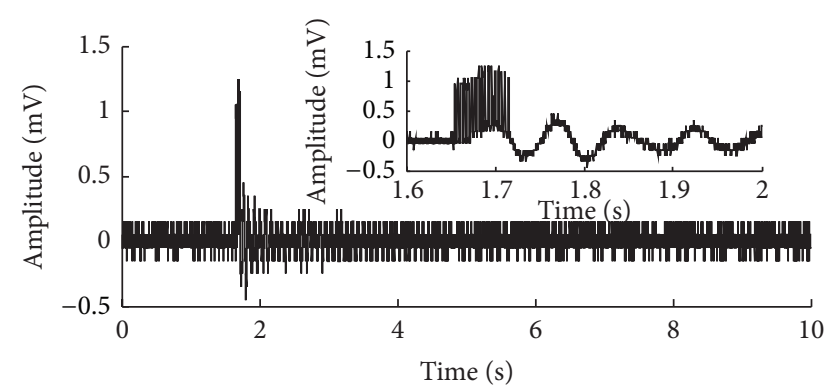

FIGURE 9: Original TEM signal obtained during coal blasting rupture.
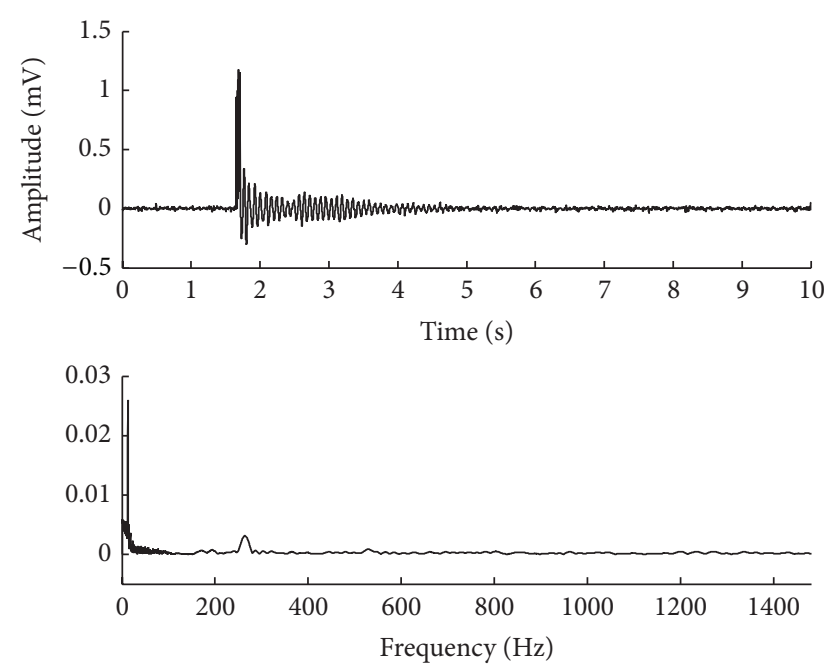

FIGURE 10: TEM signal processed by EEMD.

Comparing Figures 9 and 10, the interference component in the original signal was filtered. Its time domain feature became much clearer, and its FFT spectrum showed that the frequency of the signal was mainly focused around $0-25 \mathrm{~Hz}$. Another frequency band is around $250-280 \mathrm{~Hz}$.

From Figures 9 and 10, it can be seen that the TEM signal produced during coal blasting rupture presents two phases, a pulse rise and a tailing shock, which confirm the SHPB test result (Figure 3).

One difference from the single-pulse from the SHPB results is that the TEM signal obtained in the field performs as pulse clusters. The amplitude of the tailing shock is much higher than the SHPB results, and the duration is longer. An analysis of the 22 group signals showed that all the results coincide with the aforementioned characteristics.

\section{Low-Frequency TEM Effect Generation Mechanism Analysis and Discussion}

4.1. Mechanistic Analysis of the Pulse Part of TEM Signal. Dynamic analysis results of small-scale coal specimens tested in the SHPB suggested that, in a certain strain rate range, the dynamic response of coal changes from hardening to softening as the strain rate increasing. The peak value increased as

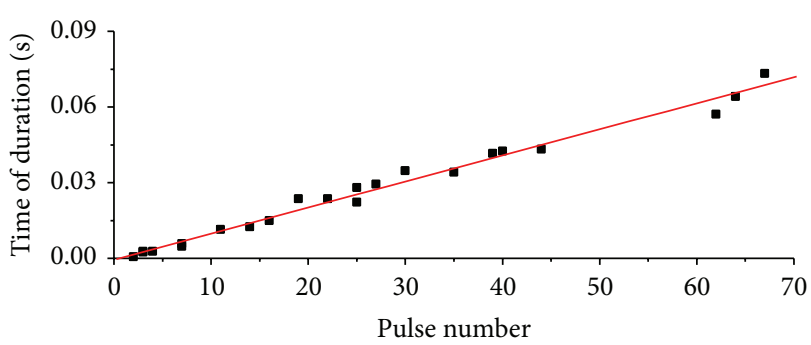

FIGURE 11: Relationship between $N_{\text {pulse }}$ and $T_{\text {duration }}$.

the impact velocity, the strain rate, and the fracture stress limit value increased.

According to the SHPB test principle [29], the incident wave reverberates in the coal specimen 9-10 times or more. The shock wave reverberation in the coal specimen produces compression and tensile deformation.

And on the basis of fractoemission theory $[30,31]$ and the theories about the strain rate sensitivity of coal $[22,32]$, with the impact loading, the stress waves compress or stretch the coal-mass; internal cracks expand quickly and will be closed and opened in this process. A concussion capacitor can be formed at the fracture surface, leading to charge separation and transfer. High concentration of electrical charge at the surface transferred instantaneously and the energy was given out transiently, in the form of electromagnetic waves.

This is considered as a significant cause for the generation of pulsed electromagnetic anomalies.

In order to explain this phenomenon in detail, both the numbers of pulses in the cluster $\left(N_{\text {pulse }}\right)$ and their duration ( $\left.T_{\text {duration }}\right)$ were calculated. A plot of the two parameters is shown in Figure 11.

Figure 11 shows that there is a good linear relationship between the pulse number and pulse duration, which indicate that the pulse duration of the cluster increases with pulse number.

Analysis suggests that a blasting hole causes coal rupture and induces a single TEM pulse, and multiple holes blasting induces pulse clusters. But, if some holes blast simultaneously, the TEM pulses will merge together.

4.2. Mechanistic Analysis of the Trailing Part of the Signal. Research has shown that when coal is deformed by dynamic loads in the SHPB, as the increase of the impact velocity is increased, the change in duration of the TEM signal increase is not obvious. As the velocity increases to a certain extent, its duration remains substantially constant. And as to the midvelocity, the trailing part is obvious, but as to the low or high velocity, the trailing part is not obvious.

Initial analysis suggests the following. (1) Since coal material is a weakly conductive, high resistivity medium, at low impact velocity, the TEM signal amplitude decays rapidly and cannot produce obvious attenuation concussion. (2) While at high velocity, coal specimens fragment very quickly. As the shock waves transportation time is very short, the detector cannot capture the attenuation signal. (3) While for medium velocity, the tailing part of the TEM signal was 
due to electromagnetic oscillation effects caused by shock stress unloading, coal crushing, crushed coal-mass friction, and the expansion of cracks produced during fragmentation rotating, vibrating, so the amplitude of the trailing part is much lower than the initial peak.

Compared with the laboratory test results, the trailing shock of the TEM signals during coal blasting is more obvious and lasts longer. Such changes may be caused by the coalrock-mass structure and the overlaying of dynamic load is caused by multiple holes blasting.

Firstly, according to the triboluminescence theory [33], the blasting shock waves propagating in the coal seam in the form of dilatation or shear waves produce compressive, tensile, and shear deformation in the coal-mass, which causes extrusion, friction, and vibration at the preexisting and fresh cracks or fissures and thus produce variable motion free charges. Energy is converted into electromagnetic wave which is radiated outward. But due to the special structural, mechanical, and electrical properties, the electromagnetic emission of coal would be at low frequency.

Secondly, due to the different impedance of coal seam and rock, blasting shock waves are reflected between roof or floor coal-rock seam, producing a planar waveguide effect or surface oscillations effect [23]. That will even induce resonance of coal-mass. Researches on coal resonance damage indicate that the natural frequency of coal is below $50 \mathrm{~Hz}$, and when the coal-mass is excited to resonance, the frequency of the electromagnetic radiation signals coincides with the excitation frequency. By the resonance effect, the TEM signal can be controlled by the variable motion of free charges generated by friction in the coal-mass. In addition, because the vibration damage lags behind the blasting damage effect and its intensity is much weaker than the latter, the TEM signal has a low-frequency trailing turbulence following the initial pulse.

4.3. Discussion on the Surroundings and the Piezoelectric Effect. In terms of usage, it is necessary to discuss the interference of the electromagnetic wave from the environments and the contribution of piezoelectric effect on the low-frequency TEM signal.

(1) As the literature [20] reported, the frequency bands of the electromagnetic environments in roadways of underground coal mines are relatively specific and high-frequency ones. And studies on EMR produced during the process of coal-rock deformation or fracture show that the frequency of EMR has a wide bandwidth. But the medium-high-frequency EMR signal is vulnerable to the effect of surrounding such as the high-frequency interference from mechanical and electrical equipment. So, the low-frequency electromagnetic technique has an advantage to avoid the surrounding interference in coal mine.

(2) The piezoelectric effect of rocks has been reported in various literatures [34]. However, the researches by Warwick et al. [35] and Maxwell et al. [36] indicated whether rocks contain piezoelectric materials or not; EMR phenomenon has been investigated. And researches by Cress et al. [37] and Zhu and Luo [38] revealed that the piezoelectric effect was not the true mechanism or not the main role of rock EMR. Considering the transient time of the TEM signal produced by the piezoelectric effect is about $10^{-5} \mathrm{~s}$ and the spectral content shifts to higher frequencies as grain size decreases, it could be concluded that the piezoelectric effect may not be the main cause of the low-frequency TEM.

There is no doubt that the low-frequency TEM is related to coal-rock deformation and has potential application for monitoring coal mine dynamic disasters.

\section{Conclusions}

(1) Transient electromagnetic radiation signals emerge during the damage of coal under dynamic load, and its wave form presents as a rising pulse and rapid dropping with a slight tailing oscillation. Its frequency ranges below $40 \mathrm{~Hz}$, and the signal duration and peak value increase with impact velocity.

(2) Coal fragments quickly while it is impacted by dynamic load, and cracks can expand rapidly, and high concentrations of free charge are generated at crack surfaces. Energy radiates outward in the form of electromagnetic waves while free charges are discharged and then form the TEM pulse. With the fragmentation of coal, the shock stress unloads quickly, and the TEM pulse decays rapidly. During the fragments rotating and translating, the emerged cracks continually expand. Accompanied with the friction between broken pieces, a low-frequency electromagnetic oscillation wave emerges. Unloading of the shock stress results in the intensity of the oscillation being much weaker than the initial pulse.

(3) Characteristics of the TEM signal produced during coal blasting agree with the SHPB test results. But affected by the multiple holes blasting, the blasting produces a pulse cluster in the initial part, and the pulse number increases with the number of holes.

(4) Affected by the blasting shock wave and the resonance caused by waveguide effects, the coal deformation lags behind the blasting damage effects, and its intensity is much weaker than the latter. Therefore, the TEM of coal blasting rupture is a low-frequency signal with a longer duration.

(5) The low-frequency TEM is related to coal deformation and has potential application for monitoring coal mine dynamic disasters.

\section{Conflict of Interests}

The authors declare that there is no conflict of interests regarding the publication of this paper.

\section{Acknowledgments}

This research is supported by National Natural Science Foundation Project $(51274206,51404277)$ and the Program of Central Universities Fundamental Research Funds from 
the Ministry of Education of China (2010YZ05, 2014QZ05). These supports are greatly acknowledged and appreciated.

\section{References}

[1] M.-C. He, H.-P. Xie, S.-P. Peng, and Y.-D. Jiang, "Study on rock mechanics in deep mining engineering," Chinese Journal of Rock Mechanics and Engineering, vol. 24, no. 16, pp. 2803-2813, 2005 (Chinese).

[2] Q. Qi and L. Dou, Theory and Technology of Rockburst, China University of Mining and Technology Press, Xuzhou, China, 2008 (Chinese).

[3] Y.-D. Jiang, Y.-S. Pan, F.-X. Jiang, L.-M. Dou, and Y. Ju, "State of the art review on mechanism and prevention of coal bumps in China," Journal of the China Coal Society, vol. 39, no. 2, pp. 205-213, 2014 (Chinese).

[4] B. T. Brady and G. A. Rowell, "Laboratory investigation of the electrodynamics of rock fracture," Nature, vol. 321, no. 6069, pp. 488-492, 1986.

[5] S. C. Langford, J. T. Dickinson, and L. C. Jensen, "Simultaneous measurements of the electron and photon emission accompanying fracture of single-crystal MgO," Journal of Applied Physics, vol. 62, no. 4, pp. 1437-1449, 1987.

[6] X. He and M. Liu, Electromagnetic Dynamics of Coal Containing Gas Destruction, China University of Mining and Technology Press, Xuzhou, China, 1995 (Chinese).

[7] V. Frid, J. Goldbaum, A. Rabinovitch, and D. Bahat, "Electric polarization induced by mechanical loading of solnhofen limestone," Philosophical Magazine Letters, vol. 89, no. 7, pp. 453463, 2009.

[8] C.-P. Lu, L.-M. Dou, A.-Y. Cao, and X.-R. Wu, "Research on microseismic activity rules in Sanhejian Coal Mine," Journal of Coal Science \& Engineering, vol. 14, no. 3, pp. 373-377, 2008 (Chinese).

[9] S. G. O'Keefe and D. V. Thiel, "A mechanism for the production of electromagnetic radiation during fracture of brittle materials," Physics of the Earth and Planetary Interiors, vol. 89, no. 1-2, pp. 127-135, 1995.

[10] V. Frid, D. Bahat, J. Goldbaum, and A. Rabinovitch, "Experimental and theoretical investigations of electromagnetic radiation induced by rock fracture," Israel Journal of Earth Sciences, vol. 49, no. 1, pp. 9-19, 2000.

[11] J. T. Dickinson, L. C. Jensen, S. Lee, L. Scudiero, and S. C. Langford, "Fracto-emission and electrical transients due to interfacial failure," Journal of Adhesion Science and Technology, vol. 8, no. 11, pp. 1285-1309, 1994.

[12] M. V. Kurlenya, A. G. Vostretsov, M. M. Pynzar, and G. E. Yakovitskaya, "Electromagnetic signals during static and dynamic loading of rock samples," Journal of Mining Science, vol. 38, no. 1, pp. 20-24, 2002.

[13] C.-W. Li, B.-J. Xie, W. Yang, and S. Wei, "Characteristics of transient magnetic nearby field in process of coal impact damage," Chinese Journal of Rock Mechanics and Engineering, vol. 31, no. 5, pp. 973-981, 2012 (Chinese).

[14] N. Djordjevic, J. Esterle, D. Thornton, and D. la Rosa, "A new approach for prediction of blast induced coal fragmentation," in Proceedings of the Mine to Mill Conference, pp. 175-181, The Australasian Institute of Mining and Metallurgy, Brisbane, Australia, 1998.

[15] V. Frid, "Rockburst hazard forecast by electromagnetic radiation excited by rock fracture," Rock Mechanics and Rock Engineering, vol. 30, no. 4, pp. 229-236, 1997.
[16] W. Enyuan, H. Xueqiu, L. Zhentang et al., "Electromagnetic radiation detector of coal or rock dynamic disasters and its application," Journal of China Coal Society, vol. 28, no. 4, pp. 366-369, 2003.

[17] S.-Q. Qian, K.-X. Ren, and Z. Lü, "Experimental study of the features of VLF, MF, HF and VHF electromagnetic radiation accompanying rock fracture," Acta Seismologica Sinica, vol. 9, no. 3, pp. 447-454, 1996.

[18] W. Enyuan, H. Xueqiu, and L. Zhonghui, Coal and Rock EMR Technology and Its Application, Science Press, Beijing, China, 2009, (Chinese).

[19] E.-Y. Wang, X.-Q. He, Z.-T. Liu, L.-M. Dou, S.-N. Zhou, and B.-S. Nie, "Electromagnetic radiation detector of coal or rock dynamic disasters and its application," Journal of the China Coal Society, vol. 28, no. 4, pp. 366-369, 2003 (Chinese).

[20] J. Sun, F. Wang, T. Pan, L. Tang, S. Wang, and Y. Ming, "Electromagnetic environments in roadways of underground coal mines and a novel testing method," Mining Science and Technology, vol. 20, no. 2, pp. 244-247, 2010.

[21] A. Rabinovitch, V. Frid, D. Bahat, and J. Goldbaum, "Decay mechanism of fracture induced electromagnetic pulses," Journal of Applied Physics, vol. 93, no. 9, pp. 5085-5090, 2003.

[22] V. M. Kosenkov and A. R. Rizun, "Characterization of the pulse destruction of black coal using the Kolsky method," Surface Engineering and Applied Electrochemistry, vol. 47, no. 2, pp. 189195, 2011.

[23] A. Rabinovitch, V. Frid, and D. Bahat, "Surface oscillations-a possible source of fracture induced electromagnetic radiation," Tectonophysics, vol. 431, no. 1-4, pp. 15-21, 2007.

[24] X.-Q. He, B.-S. Nie, E.-Y. Wang, L.-M. Dou, M.-J. Liu, and Z.-T. Liu, "Electromagnetic emission forecasting technology of coal or rock dynamic disasters in mine," Journal of the China Coal Society, vol. 32, no. 1, pp. 56-59, 2007 (Chinese).

[25] G. Tianbao, Coal Experiment Study on Forced Vibration Response and Fracture Characteristics, China University of Mining and Technology, Beijing, China, 2013, (Chinese).

[26] E. D. H. Davies and S. C. Hunter, "The dynamic compression testing of solids by the method of the split Hopkinson pressure bar," Journal of the Mechanics and Physics of Solids, vol. 11, no. 3, pp. 155-179, 1963.

[27] X. J. Wu and D. A. Gorham, "Stress equilibrium in the split Hopkinson pressure bar test," Le Journal de Physique IV, vol. 7, no. C3, pp. 91-96, 1997.

[28] Z. Wu and N. E. Huang, "Ensemble empirical mode decomposition: a noise-assisted data analysis method," Advances in Adaptive Data Analysis, vol. 1, no. 1, pp. 1-41, 2009.

[29] C. Bacon, "An experimental method for considering dispersion and attenuation in a viscoelastic hopkinson bar," Experimental Mechanics, vol. 38, no. 4, pp. 242-249, 1998.

[30] J. T. Dickinson, "Fractoemission," in Encyclopedia of Materials: Science and Technology, K. H. J. Buschow, R. W. Cahn, M. C. Flemings et al., Eds., pp. 3254-3257, Elsevier, 2001.

[31] J. T. Dickinson, C. L. Jensen, and A. Jahan-Latibari, "Fractoemission: the role of charge separation," Journal of Vacuum Science \& Technology A, vol. 2, no. 2, pp. 1112-1116, 1984.

[32] J. R. Klepaczko, "On the role of microcracking inertia in rate sensitivity of coal at high strain rates," Archives of Mechanics, vol. 40, no. 4, pp. 345-358, 1988.

[33] A. J. Walton, “Triboluminescence," Advances in Physics, vol. 26, no. 6 , pp. 887-948, 1977. 
[34] U. Nitsan, "Electromagnetic emission accompanying fracture of quartz-bearing rocks," Geophysical Research Letters, vol. 4, no. 8, pp. 333-336, 1977.

[35] J. W. Warwick, C. Stoker, and T. R. Meyer, "Radio emission associated with rock fracture: possible application to the great Chilean Earthquake of May 22, 1960," Journal of Geophysical Research, vol. 87, no. 4, pp. 2851-2859, 1982.

[36] M. Maxwell, R. D. Russell, A. W. Kepic, and K. E. Butler, "Electromagnetic responses from seismically excited targets B: non-piezoelectric phenomena," Exploration Geophysics, vol. 23, no. 2, pp. 201-208, 1992.

[37] G. O. Cress, B. T. Brady, and G. A. Rowell, "Sources of electromagnetic radiation from fracture of rock samples in the laboratory," Geophysical Research Letters, vol. 14, no. 4, pp. 331334, 1987.

[38] Y.-Q. Zhu and X.-L. Luo, "A study of mechanism on electromagnetic emission associated with rock fracture," Acta Geophysica Sinica, vol. 34, no. 5, pp. 594-601, 1991 (Chinese). 

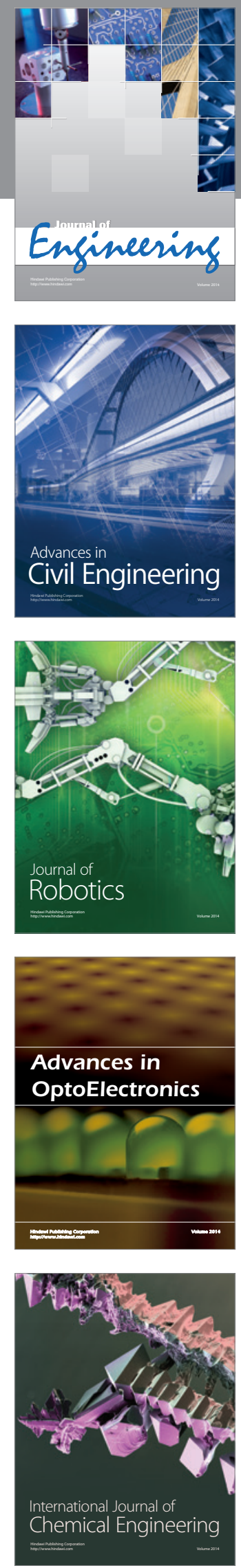

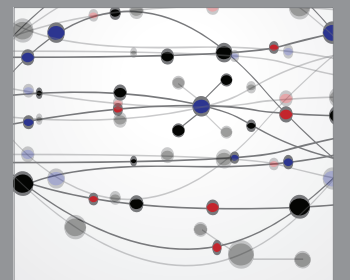

The Scientific World Journal
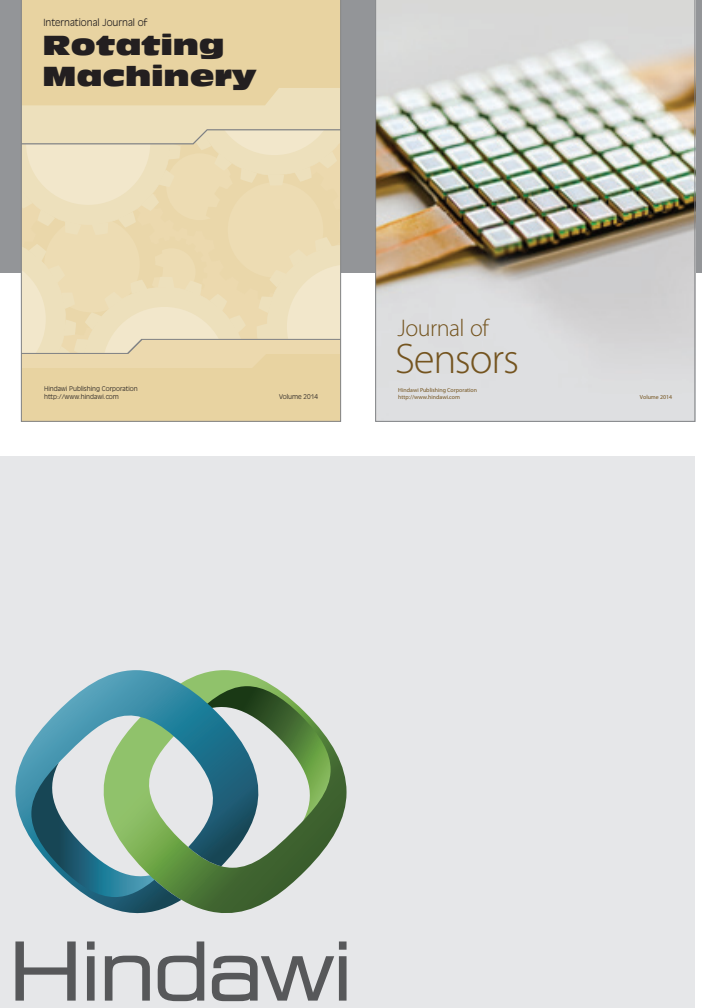

Submit your manuscripts at http://www.hindawi.com
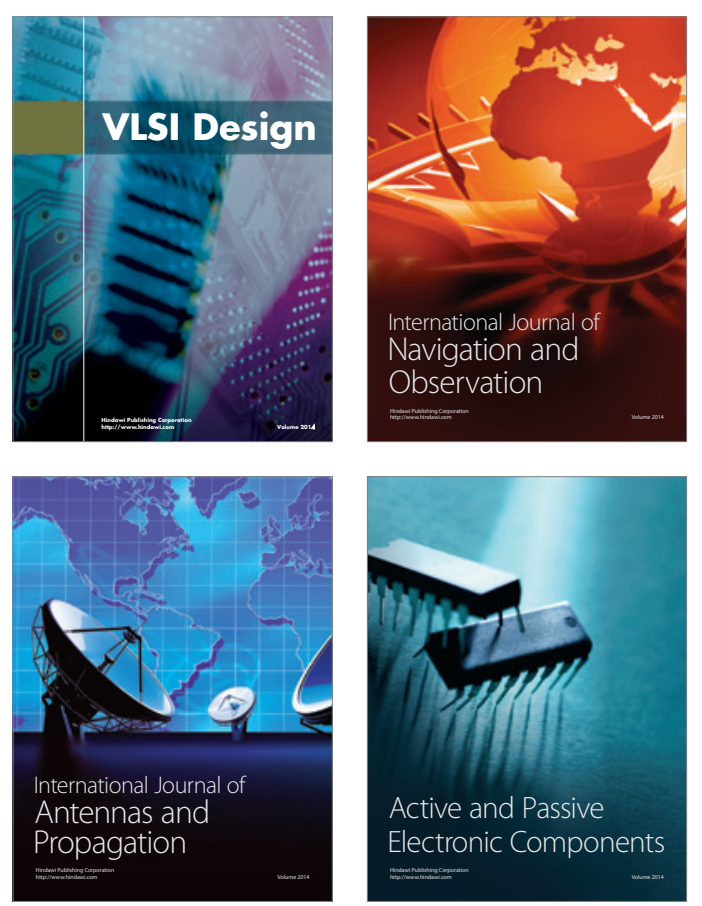
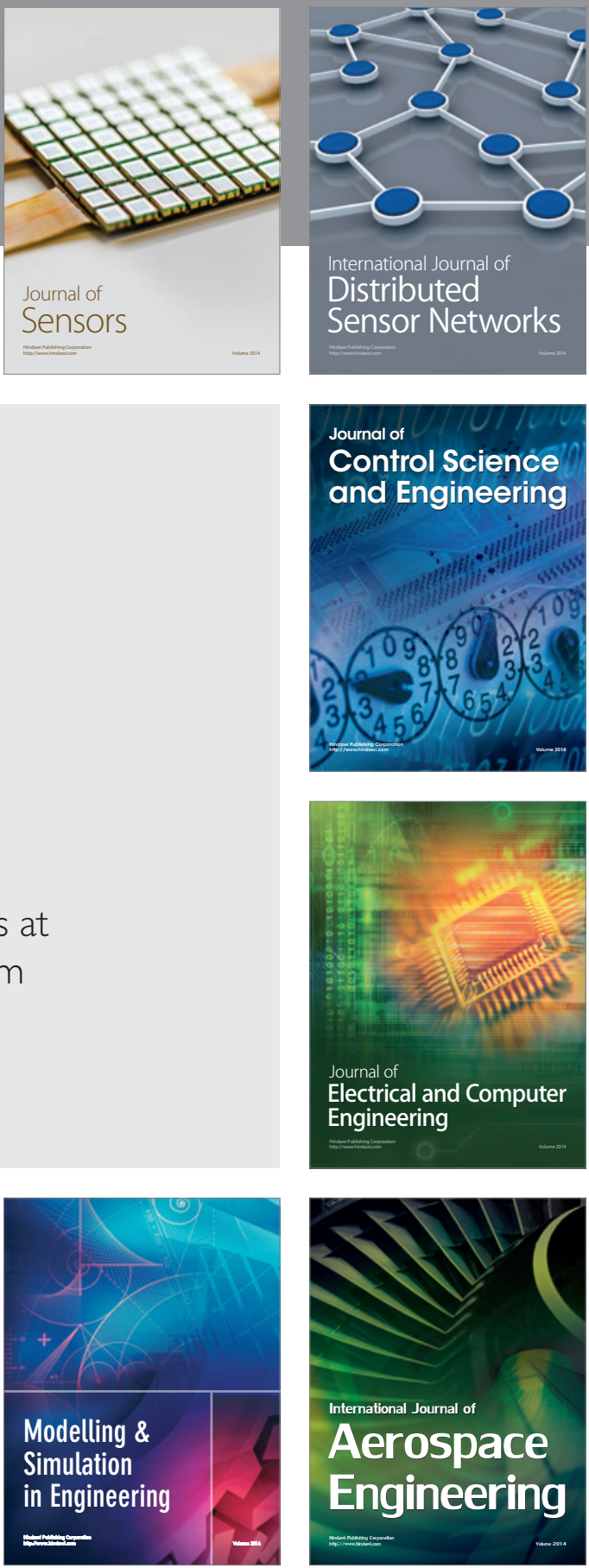

Journal of

Control Science

and Engineering
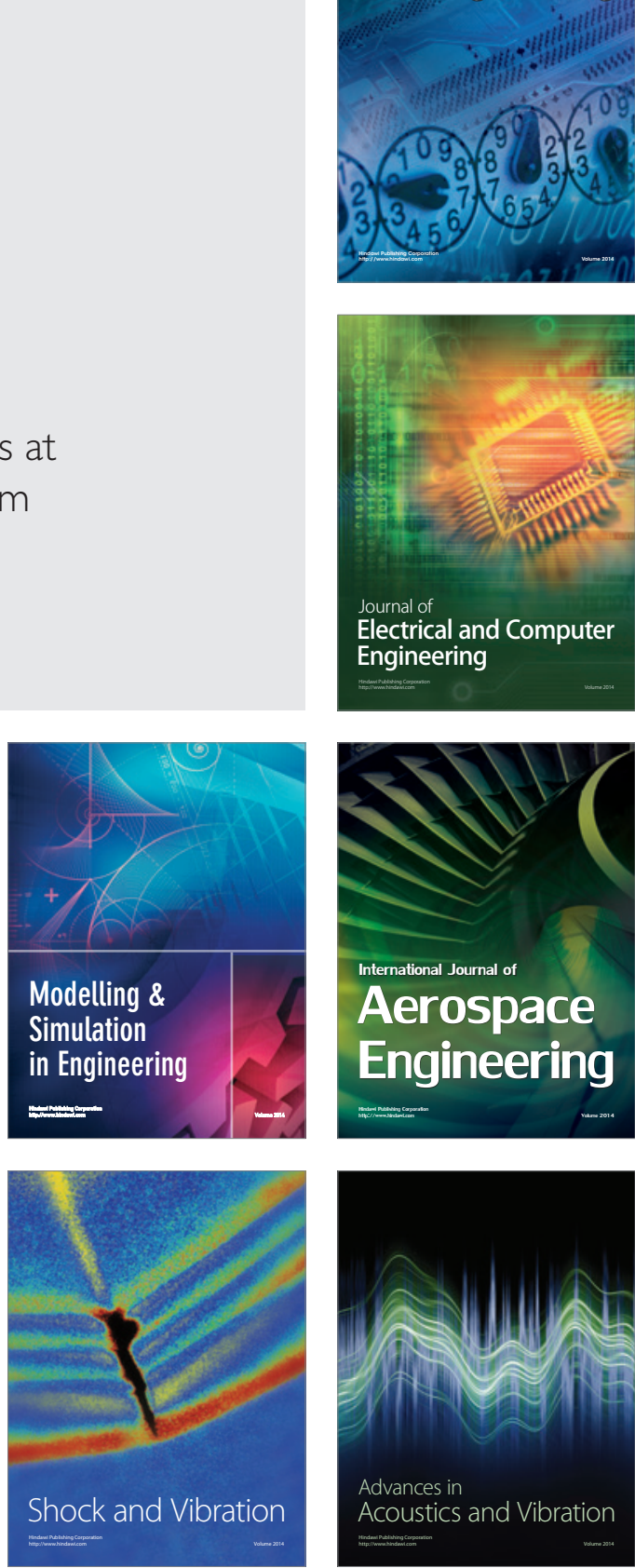